\title{
FOCUSING TYPE HIGH POWER TRANSDUCER USING BURST DRIVE FOR ULTRASONIC WELDING
}

\author{
Minoru Kurosawa1, Katsuya Nakazawa, Yoshikazu Koike and Sadayuki Ueha \\ Precision and Intelligence Laboratory, Tokyo Institute of Technology \\ Nagatsuta, Midori-ku, Yokohama 227, JAPAN
}

\begin{abstract}
In order to operate ultrasonic welding tools more efficiently at high frequency over $100 \mathrm{kHz}$ to $\mathrm{MHz}$ order, a focusing type transducer is proposed. The transducer is constructed with metal bodies. A disk PZT element is glued on the back surface of one metal. The PZT is driven by burst waves with an electric source. The metal bodies consist two kinds of materials for transmission medium. The intermediate boundary of the two metals is formed spherical so that the plain wave from the PZT element concentrates with refraction due to the difference of the propagation velocity of the dilatational wave. At this concentrated region, the transducer has the output mechanical port so that a work is placed here. A driving frequency of the $P Z T$ is a resonance frequency of the thickness mode of the element. A transduce efficiency from electricity to acoustic power is about $90 \%$ calculated from a simple model. For designing this transducer, numerical simulation using FEM and Newmark- $\beta$ method is carried out. This simulation shows that the transform gain of the vibration velocity is about four times and amplitude distribution at the output surface is almost uniform at $570 \mathrm{kHz}$. The experiment has supported this result. It is available to obtain $1.2 \mathrm{~m} / \mathrm{s}$ vibration velocity at the output.
\end{abstract}

\section{OPERATION OF THE TRANSDUCER}

If the dimension of a transducer is lager than a wave length, the vibration mode of the transducer is rather complicated[1],[2]. Therefor, the design of the vibration system with high vibration velocity amplification becomes difficult. Introduction of noncontinuous drive is one solution to avoid this kind of problem. Because intermittent drive suppresses the excitation of the complicated mode distribution caused by standing wave in the disk transducer. On this account, a burst drive is employed instead of continuous drive[3].

In the case of the burst drive of the disk PZT element, we can expect the piston-like vibration of the plane surfaces at its thickness resonant frequency. This vibration mode is proper for concentration by using acoustical lens constructed in a wave guide. The complex distribution vibration mode is not suitable for focusing type transducer.

Propagating plane wave can be concentrated with a boundary of two kind of material due to the refraction. This phenomenon is applied to a new type high power transducer as shown in Fig. 1. The burst driven PZT element transmits the several trained plane wave in copper. The boundary between copper and steel has a shape of rotational hyperbolic surface. Because of the difference of the propagating velocity of dilatational wave, the plane wave transmitted from the PZT would be refracted at the boundary. The refraction causes the power concentration at a focal position as shown in the figure.

This kind of acoustical lens has already been applied in several application such as ultrasonic micro scopes and so on. In such cases, however, the boundary consists of solid and liquid. In actual application for high power field, solid and solid

1 currently Dept. of Precision Machinery Engineering, Faculty of Engineering, University of Tokyo 
component is superior.

\section{BEHAVIOR OF THE PZT DISK}

We used a disk PZT element which has $60 \mathrm{~mm}$ diameter and $3.5 \mathrm{~mm}$ thick (NGK Spark Plug Co. ; MT18). The electrical frequency response by continuous drive is shown in Fig. 2. Obviously, in the vicinity of the thickness resonance mode, there are many numbers of radial resonance modes. These radial modes distort the piston-like vibration mode of the thickness resonance as shown in Fig. 3. The distribution of at the output surface is not good for power application.

In the case of the transient burst drive, the vibration distribution is improved as shown in Fig. 4. The vibration velocity was measured by using laser Doppler vibrometer without mechanical load. The vibration distribution was synthesized from the transient measured data at each point. First several cycles, the vibration mode is similar to the piston motion.

The limit of the vibration velocity was measured to know the possibility of the output power. The maximum vibration velocity was about $0.3 \mathrm{~m} / \mathrm{s}$ as shown in Fig. 5. From this value and the specific impedance of the copper, the output maximum power is about $5 \mathrm{~kW}$. The efficiency of the transducer depends on the matching condition. From the calculation using the measured values about the PZT element and the specific impedance of the copper, the efficiency was estimated to be about $90 \%$. This value seems to be high enough.

\section{DESIGN OF THE WAVE GUIDE}

If the aperture of the lens is much larger than the wave length, the focal point is fixed geometrically. For the first trial, however, we used $570 \mathrm{kHz}$ and 60 $\mathrm{mm}$ aperture diameter, so that we could not apply this approximation. The wave length was about $10 \mathrm{~mm}$ and the aperture diameter of the lens was $60 \mathrm{~mm}$. From the direct integral of the boundary surface, the focal point was investigated. The focal point is found out from the Fig. 6 . It was about $20 \mathrm{~mm}$, although the designed focal length was $100 \mathrm{~mm}$.

The transmission efficiency at the boundary of the two medium was examined. The boundary is not orthogonal against the propagation direction. Therefore we should consider the mode conversion here. The transmission efficiency from the dilatational wave to dilatational wave through the boundary was calculated as shown in Fig. 7. In the case of $f=100$, the efficiency was lager than $96 \%$.

The wave propagation was simulated numerically to design the wave guide. The FEM and Newmark- $\beta$ method $[4]$, one of the backward difference method, were used to carry out the 3 dimensional time domain simulation[5]. The dimensions of the transducer are indicated in Fig. 8. The output vibration distribution was simulated as shown in Fig. 9. The flatness of the vibration distribution was rather good and the transform ratio was about 4 .

\section{EXPERIMENTS}

The experimental results are shown in Figs. 10, 11 and 12. The vibration mode of the output surface shown in Fig. 10 was piston-like as predicted in the simulation indicated in Fig. 9. The time-response of the back surface of the PZT and the output surface as in Fig. 11 show that the transform of the vibration amplitude is about 4 . The output vibration velocity was increased by changing the driving surface amplitude as shown in Fig. 12.

Because of the insufficient ability of the power amplifiers the resultant output power was not so intensive. We require much lager power to obtain some interesting effect on ultrasonic welding.

The gain of the wave guide was about 4 . In order to obtain lager ratio, we should enlarge the aperture size by increasing the dimension or the frequency.

\section{CONCLUSION}

A focusing type high power transducer using burst drive of the PZT element of its thickness mode is proposed. The numerical simulation of the transducer was successfully carried out. The experimental results supported it.

\section{REFERENCE}

[1] K. Adachi and S. Ueha, "Modal vibration control of large ultrasonic tools with the use of wave-trapped horns," J. Acoust. Soc. Am., 87(1), pp.208-214(1990).

[2] K. Adachi and S. Ueha, "Modal vibration control of large ultrasonic tools in high amplitude operation," Jpn. J. Appl. Phys., 28(2), pp.279-286(1989).

(3) K. Nakazawa, M. Kurosawa, Y. Koike and S. Ueha, "Focusing type high power transducer using burst 
drive," Tech. Rep. of IEICE, US92-44(1992-09).

[4] N. M. Newmark, "A method of computation for structural dynamics," J. Eng. Mech. Div., ASCE, 85, pp.67-94(1959).

[5] R. Ludwig, and W. Lord, "A finite-element formulation for the study of ultrasonic NDT system," IEEE Trans. on Sonics. Ultrason., 35, pp.809-820(1988).

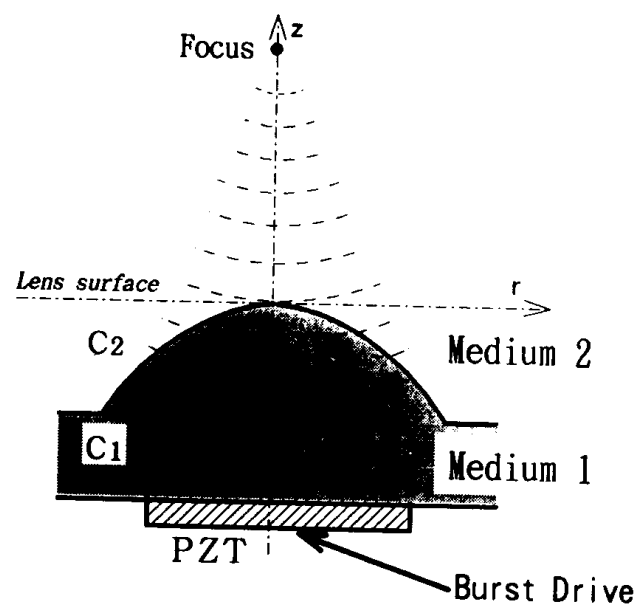

Fig. 1 Principle of a focusing transducer.

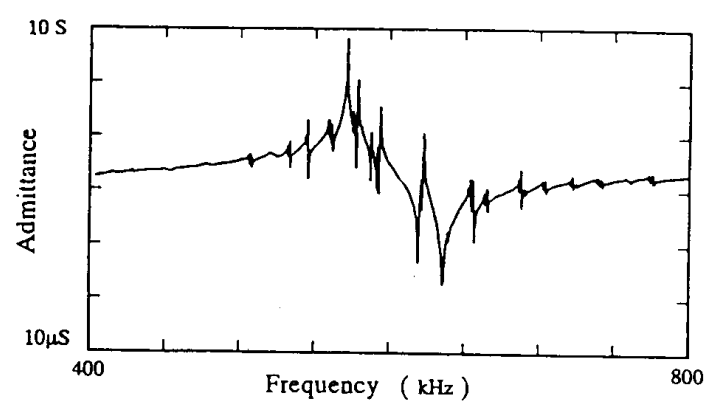

Fig. 2 Admittance of the PZT element.

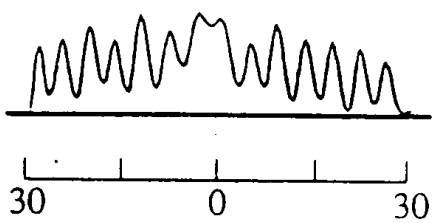

Distance from center (mm)

Fig. 3 Vibraiton mode of the PZT.

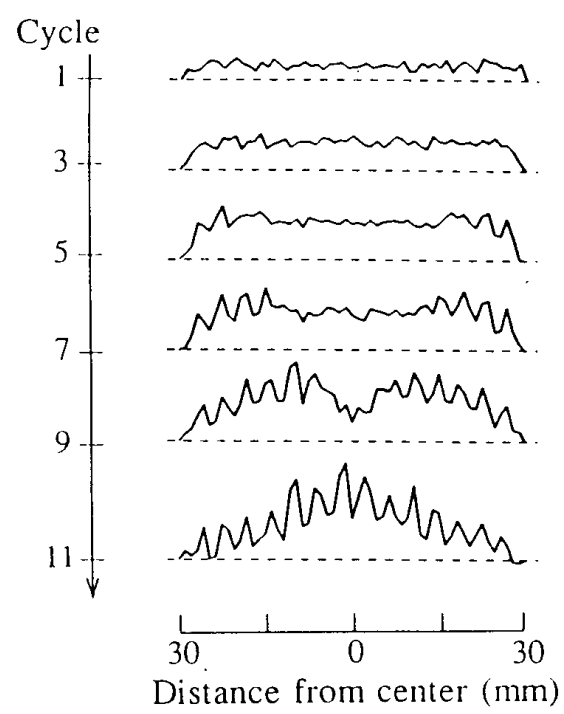

Fig. 4 Vibration velocity distribution of PZT surface with burst drive.

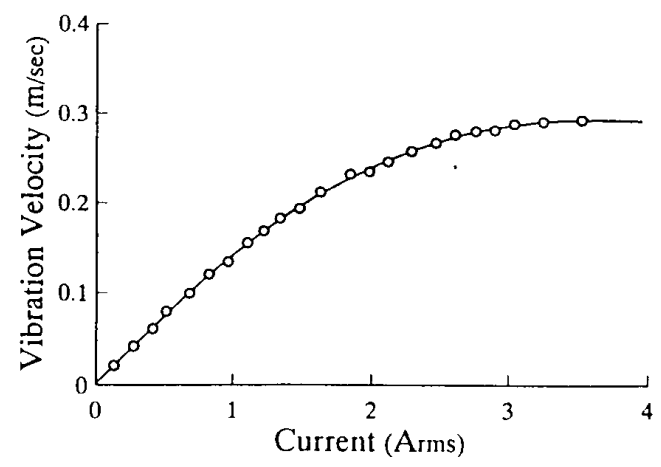

Fig. 5 The vibration velocity of the PZT surface.

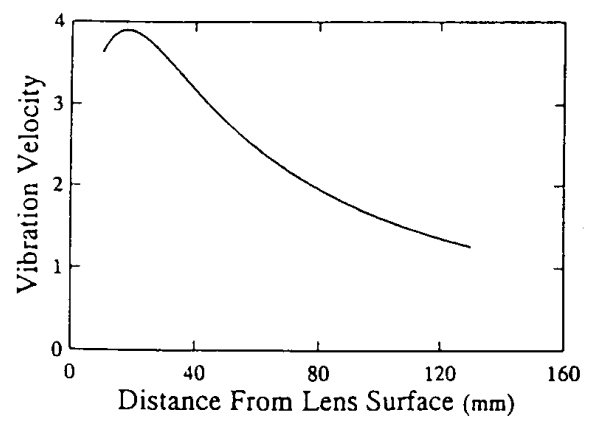

Fig. 6 Vibration velocity distribution along $\mathrm{z}$ axis. 


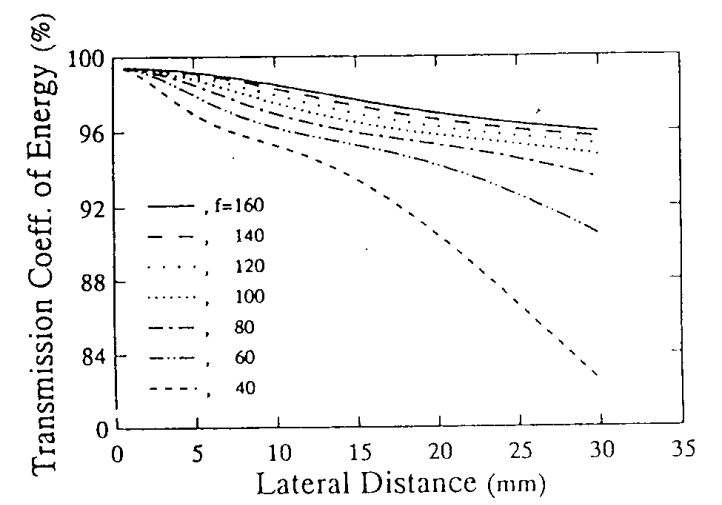

Fig. 7 Transmission energy through the boundary.

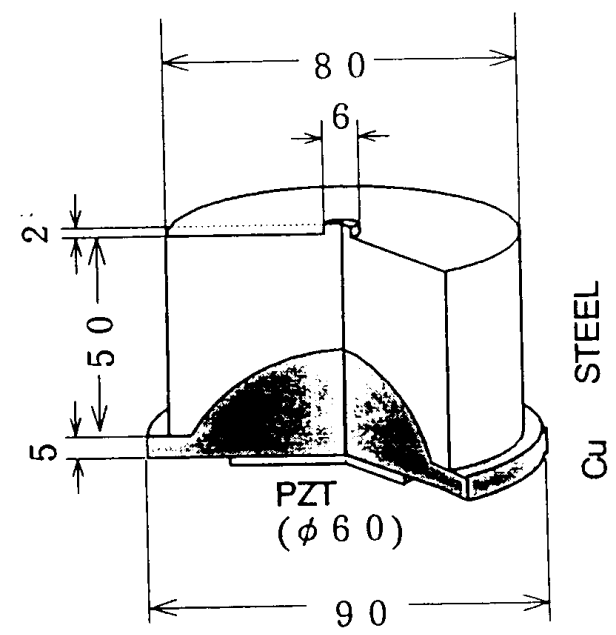

Fig. 8 Schema of the focusing transducer.

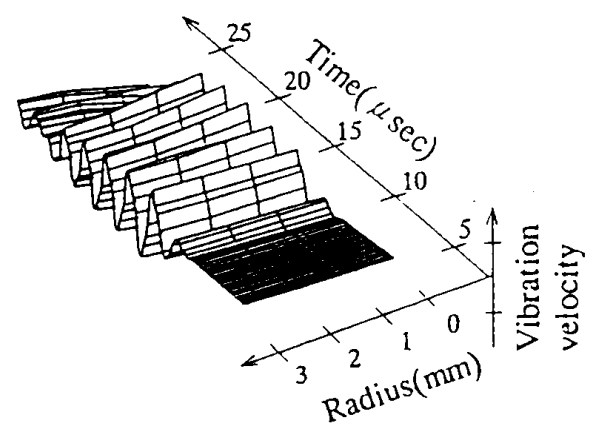

Fig. 9 Vibration velocity distribution at output surface by simulation.

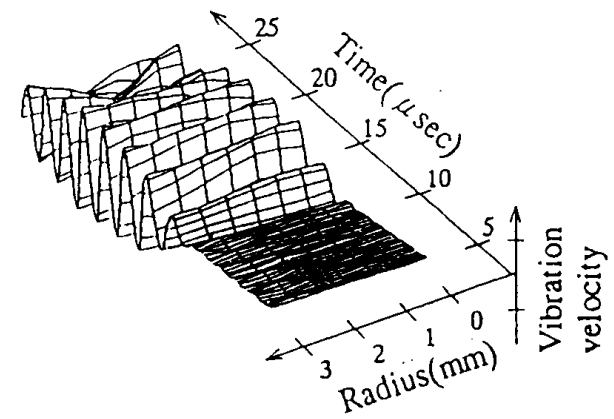

Fig. 10 Vibration velicity distrubution at output surface by experiment.

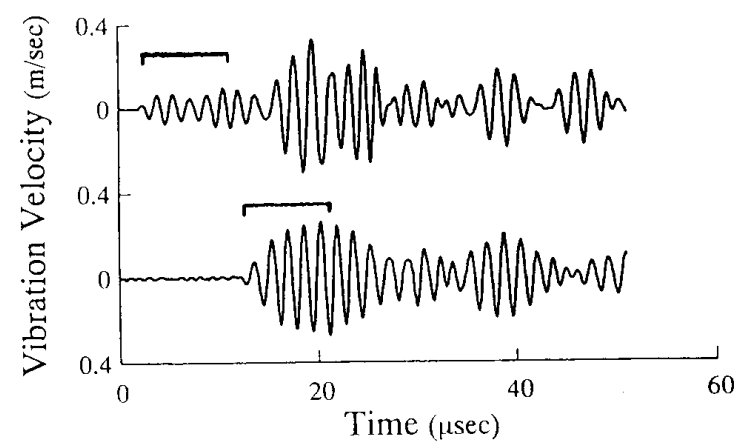

Fig. 11 Vibration velocity : upper, PZT surface ; lower, output surface.

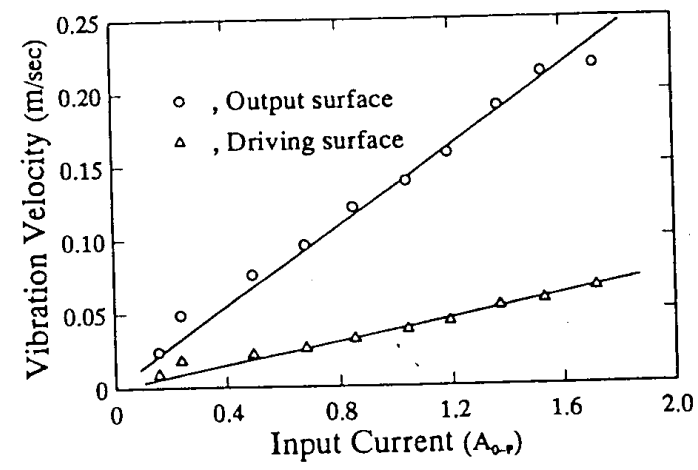

Fig. 12 Step up ratio of the transducer. 